\title{
Agriculture based plant leaf health assessment tool: A Deep Learning perspective
}

\author{
${ }^{1}$ Syed Inthiyaz, ${ }^{2}$ M V D Prasad, ${ }^{3}$ R. Usha Sri Lakshmi, ${ }^{4}$ N.T.B. Sri Sai, ${ }^{5}$ P. Pavan Kumar, ${ }^{6}$ Sk Hasane Ahammad \\ Associate professor ${ }^{1,2,6}$, B. Tech ${ }^{3,4,5}$, \\ 1,2,3,4,5,6 Department of ECE, Koneru Lakshmaiah Education Foundation, Vaddeswaram, Guntur.
}

\begin{abstract}
For the most part, mango leaves principally get influenced by three basic sicknesses they are Anthracnose, Bacterial canker, Powdery mildew. The previously mentioned infections influence the development of mango tree, decline the life expectancy and decrease the natural product creation. Thinking about this we were doing the task under the territory of leaf malady characterization. The fundamental point of our venture is to arrange the state of the leaf, needs to recognize the malady from which it is experiencing. Thus, it will be for the most part helpful for ranchers keeping from crop misfortune financially. They can kill the illness in the underlying state itself. We were utilizing the profound learning system to recognize the tainted leaves. We propose a CNN single-stream model to order the picture. Our informational collection comprises a sum of 800 pictures arranged into two kinds, the main sort comprises a preparation set and the subsequent kind comprises of the testing set. Preparing comprises of 150 pictures and testing comprises of 50 pictures in every envelope.
\end{abstract}

Key words: CNN, Max-pooling, Convolution, Soft-max, dense layers.

\section{INTRODUCTION}

Picture classification is an energetic region of research in picture comprehension and PC vision. Inexhaustible classifiers have been proposed in writing for various applications. In this paper, we propose to utilize a portion of these models of feature extraction and classification. Fungal illnesses are normal in plant leaves. The illnesses in the plants are caused by dropping the quality and the amount of the horticulture production. The plant infections influence the nature of the leaves, organic products, stem, vegetables, and their items. This vigorously impacts efficiency and reflects against the price. Food and Agricultural Organization evaluated that the total populace will reach nine billion in the upcoming years, in this manner requiring about seventy percent development in the nourishment generation for a relentless inventory. The illnesses are the biotic variables that are either brought about by the organisms, microbes or green growth though, disarranges are the abiotic components brought about by the temperature, precipitation, supplement insufficiency, dampness and so forth. Consequently, in the field of agriculture, identification of infection in plants assumes a significant job. To distinguish a plant ailment in the extremely introductory stage, utilization of programmed sickness discovery strategy is valuable. For example, illnesses named are an Anthracnose ailment, powdery mildew, bacterial canker found in the mango tree. In such situations, early location could have been productive. Automatic detection of the diseases is cheaper in cost and just by seeing the diseased symptoms on the plant leaves, detection becomes simple. The proposed CNN strategy is programmed, computationally it is proficient. Along these lines, this work proposes a profound learning procedure named as CNN for the arrangement of leaves contaminated by bacterial canker, anthracnose, and powdery mildew. Deep Learning can be comprehended as a calculation that is made out of concealed layers of different neural systems. It takes a shot at solo information and is known to give precise outcomes than conventional calculations. As it goes further and more profound, it channels the intricate highlights and consolidates with those of the last layer, in this manner better outcome. Profound learning structures, for example, profound neural systems, profound conviction systems, repetitive neural systems and convolutional neural systems have been applied to fields including PC vision, discourse acknowledgment, normal language handling, sound acknowledgment, informal organization sifting, machine interpretation, bioinformatics, medicate plan, therapeutic picture examination, material review and table top game projects, where they have delivered results similar to and at times better than human specialists.

\section{LITERATURE REVIEW}

In [1] the deep learning approach that depends on improved convolutional neural systems for the constant discovery of apple leaf sicknesses. Advances utilized are information expansion and picture comment. Models utilized are GoogLeNet Inception structure, Rainbow link, R-CNN, VGG-INCE, AlexNet, INAR-SSD are utilized. Generating the apple leaf malady image annotation and data augmentation then the detection by the single-shot multi-box detector. Structure of origin module, VGG-INCEP network model is for the most part utilized. In the exploratory assessment, a trial arrangement is done, the informational index of 26,377 
pictures are gathered. Examination of pre-organize acknowledgment precision, disarray lattice, result correlation of different location calculations, information expansion examination tests, highlight representation process, exactness versus speed, identification representation, and disappointment investigation are prepared. Alternaria leaf spot, grey spot, brown spot, rust, mosaic is five normal sorts of apple leaf ailments that seriously influence apple yield. The outcome is broke down as the INAR-SSD model understands a location execution of $78.80 \% \mathrm{mAP}$ on ALDD, with a high-detection speed of 23.13 FPS of the sickness contaminated to the apple leaf.

In [2] the field of farming data, automatic identification, and conclusion of maize leaf illnesses are finished. Models utilized are improved GoogLeNet, Cifar10 dependent on profound getting the hang of, pooling blends, dropout tasks, and rectified straight unit capacities are done. Dataset is gathered, the growth strategy is utilized, picture pre-handling and marking ought to be finished. Convolutional neural systems procedure will be finished. Steps of the stream are convolution, initiate the capacity, pooling, dropout, misfortune work, hyper parameters to be determined from the given informational index. Results are taken from the tested examination for the given dataset. Nine kinds of maize leaves are recognized by utilizing profound $\mathrm{CNN}$, the exactness rate is acquired with $98.8 \%, 98.9 \%$ for the two models.

\section{METHODOLOGY}

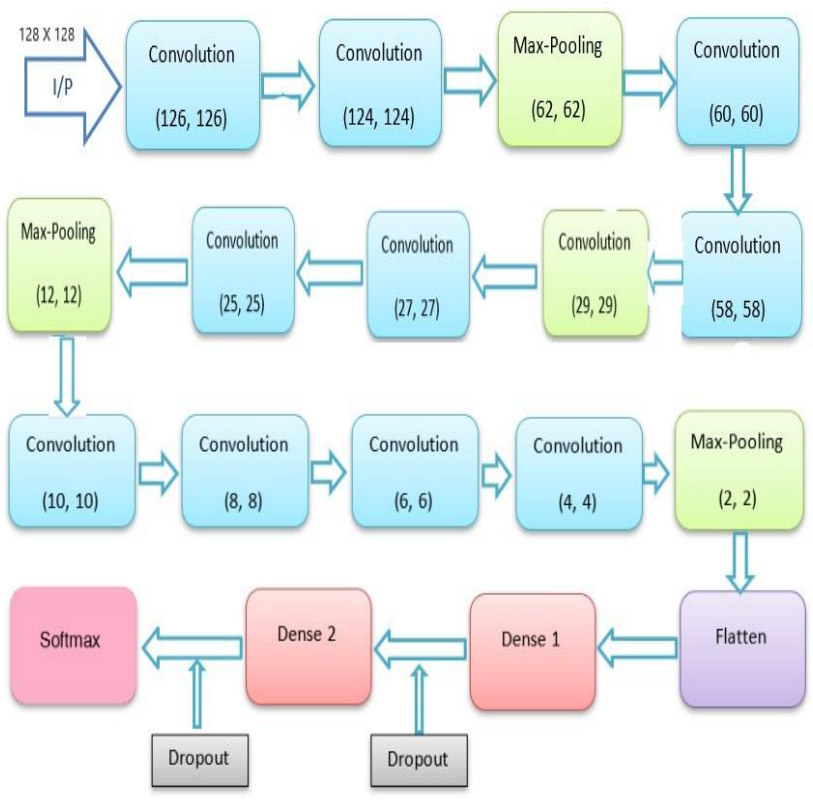

Figure 1: Block diagram

Fig 1: This block diagram explains the layers associated with the project. The information is given as a picture of size $128 * 128$ after the convolution layer the yield will be $126 * 126$. The convolution layer will extract in the form of a matrix. Again convolution layer happens and the size of the picture gets $124 * 124$. Convolutional layers apply convolution operation to the information, passing the outcome to the following layer. The convolution copies the reaction of an individual neuron to visual boosts. In the maximum pooling layer, the most well-known sort of pooling is a pooling layer of filter size $(2,2)$ utilizing the MAX activity. It would take the limit of each $4 * 4$ grid of the first picture. Again convolution is applied to the came about sign then the yield gets $60 * 60$, maxpooling is applied the yield gets $12 * 12$. Again 4 layers of convolution are applied and the yield gets $4 * 4$. At that point, max-pooling is applied yield gets $2 * 2$. It is levelled and goes through the dense layers and the dropout layer is included for each thick layer. In conclusion, it is given to the soft-max layer. The soft-max work computes the probabilities of each target class's overall conceivable objective classes. Later the determined probabilities will be useful for determining the target class for the given source inputs. The principle bit of leeway of utilizing Soft-max is the output probabilities range. The range will be from 0 to 1 , and the whole of the considerable number of probabilities will be equivalent to one.

\section{DATASET OF MANGO LEAVES COLLECTED IN OUR UNIVERSITY}

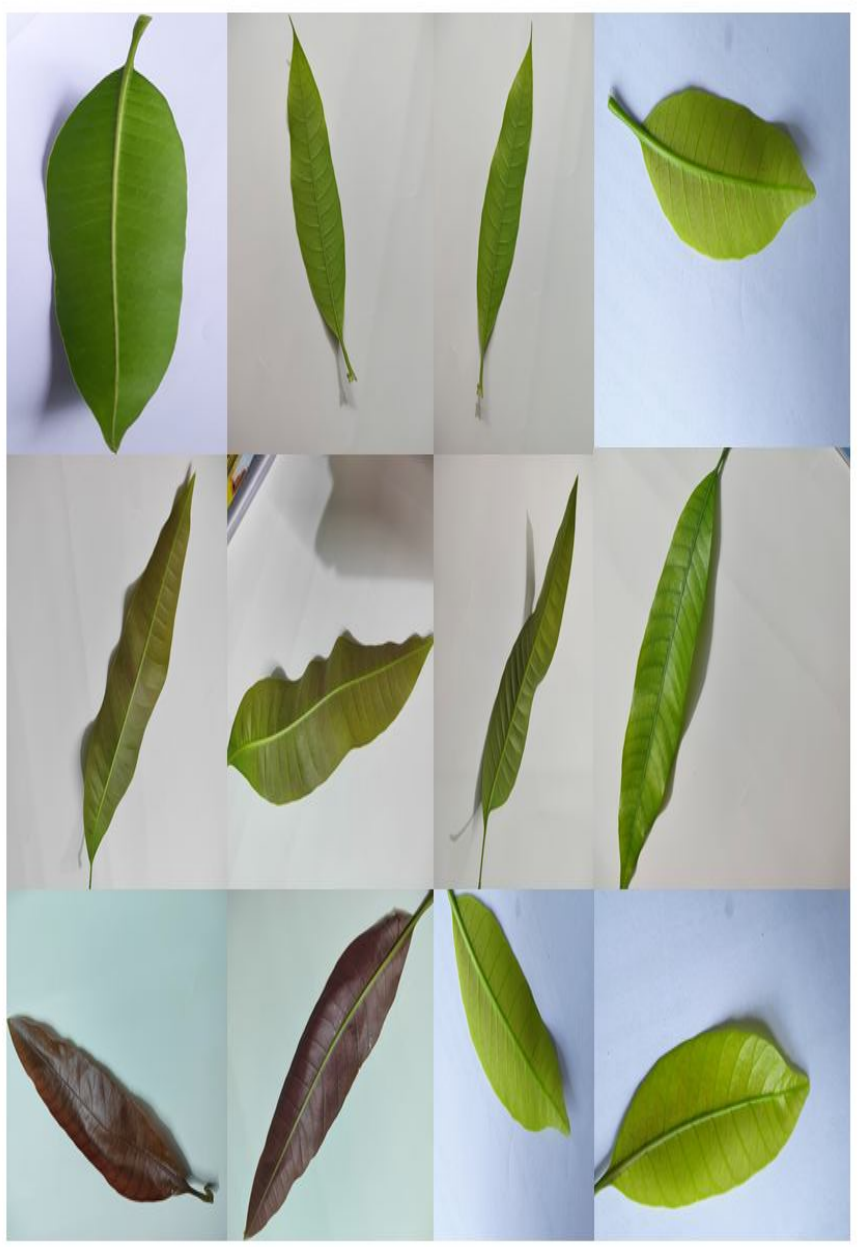

Figure 2: Healthy dataset collected in KL (Deemed to be university) 
Table 2: Table for recognition rates

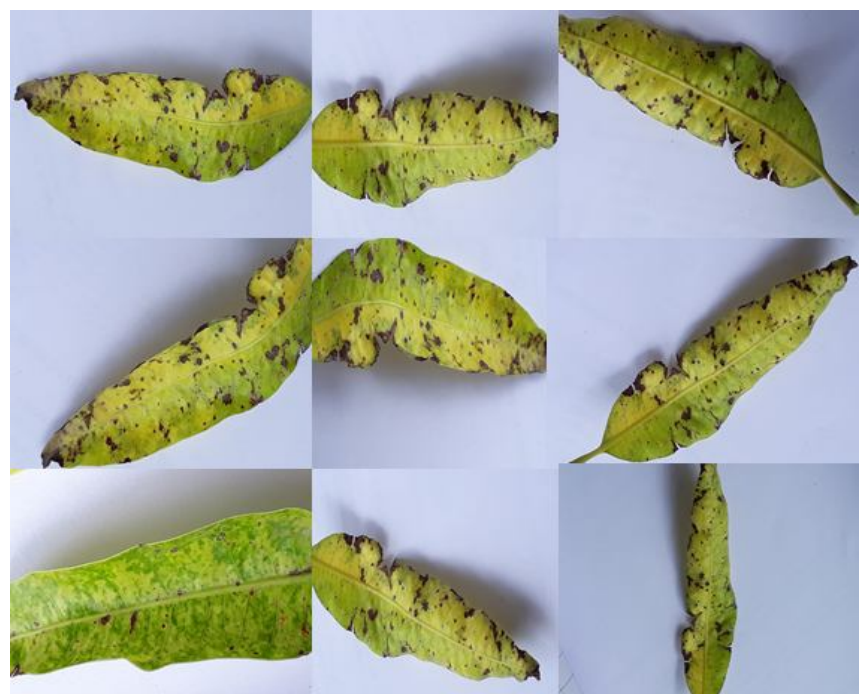

Figure 3: Powdery Mildew dataset collected in KL (Deemed to be university)

Figures 2,3,4,5: The training and testing dataset of 800 pictures of mango leaves were collected in our university. In these pictures we can see the infected leaves and healthy leaves.

Table 1: Layer information and parameters of CNN

\begin{tabular}{lll}
\hline Layer(type) & Function & Output Shape \\
\hline conv2d_1 (Conv2D) & Convolution & $8 \times 126 \times 126$ \\
activation_1 (Activation) & Activation & $8 \times 126 \times 126$ \\
conv2d_2 (Conv2D) & Convolution & $8 \times 124 \times 124$ \\
max_pooling2d_1 (MaxPooling2) & MaxPooling & $8 \times 62 \times 62$ \\
conv2d_3 (Conv2D) & Convolution & $16 \times 60 \times 60$ \\
conv2d_4 (Conv2D) & Convolution & $16 \times 58 \times 58$ \\
max_pooling2d_2 (MaxPooling2) & MaxPooling & $16 \times 29 \times 29$ \\
conv2d_5 (Conv2D) & Convolution & $32 \times 27 \times 27$ \\
conv2d_6 (Conv2D) & Convolution & $32 \times 25 \times 25$ \\
max_pooling2d_3 (MaxPooling2) & MaxPooling & $32 \times 12 \times 12$ \\
conv2d_7 (Conv2D) & Convolution & $64 \times 10 \times 10$ \\
conv2d_8 (Conv2D) & Convolution & $64 \times 8 \times 8$ \\
conv2d_9 (Conv2D) & Convolution & $64 \times 6 \times 6$ \\
conv2d_10 (Conv2D) & Convolution & $64 \times 4 \times 4$ \\
max_pooling2d_4 (MaxPooling2) & MaxPooling & $64 \times 2 \times 2$
\end{tabular}

Table 1 clarifies the number of channels we have utilized for each layer processing and how the image layers are differing by separating every one of the highlights of the picture. Numerically we have completed ten times of convolution which is two dimensional, max-pooling of four times and an activation function. The order of processing an image is convolution, activation function which includes Re-Lu function followed by max-pooling.

\begin{tabular}{|c|c|c|c|c|c|c|}
\hline Classifier & \multicolumn{6}{|c|}{ Recognition Rates (\%) } \\
\hline & \multicolumn{2}{|c|}{ Batch-I Training } & \multicolumn{2}{|c|}{ Batch-II Training } & \multicolumn{2}{|c|}{ Batch-III Training } \\
\hline & \multicolumn{2}{|c|}{ Testing with } & \multicolumn{2}{|c|}{ Testing with } & \multicolumn{2}{|c|}{ Testing with } \\
\hline & $\begin{array}{l}\text { Same } \\
\text { data }\end{array}$ & $\begin{array}{l}\text { Different } \\
\text { data }\end{array}$ & Same data & $\begin{array}{l}\text { Different } \\
\text { data }\end{array}$ & $\begin{array}{l}\text { Same } \\
\text { data }\end{array}$ & $\begin{array}{l}\text { Different } \\
\text { data }\end{array}$ \\
\hline ANN & 76.39 & 74.26 & 78.47 & 75.29 & 79.13 & 77.42 \\
\hline Deep ANN & 84.32 & 82.16 & 86.92 & 83.49 & 88.6 & 84.58 \\
\hline Our & & & & & & \\
\hline $\begin{array}{l}\text { Proposed } \\
\text { CNN(Single } \\
\text { stride) }\end{array}$ & 96.72 & 91.09 & 93.24 & 90.62 & 97.35 & 93.22 \\
\hline
\end{tabular}

Table 2 clarifies the order of three classes i.e. ANN, deep ANN, our proposed structure of CNN. For finding out the recognition rates percentage of the diseased leaves, we had performed three batches of the data for three different diseases involved in mango leaves. We tested the information with our preparation data and different data. The accuracies obtained are mentioned in the table accordingly. We had got the correctness's of ANN and profound ANN from the online sources.

\section{RESULTS}
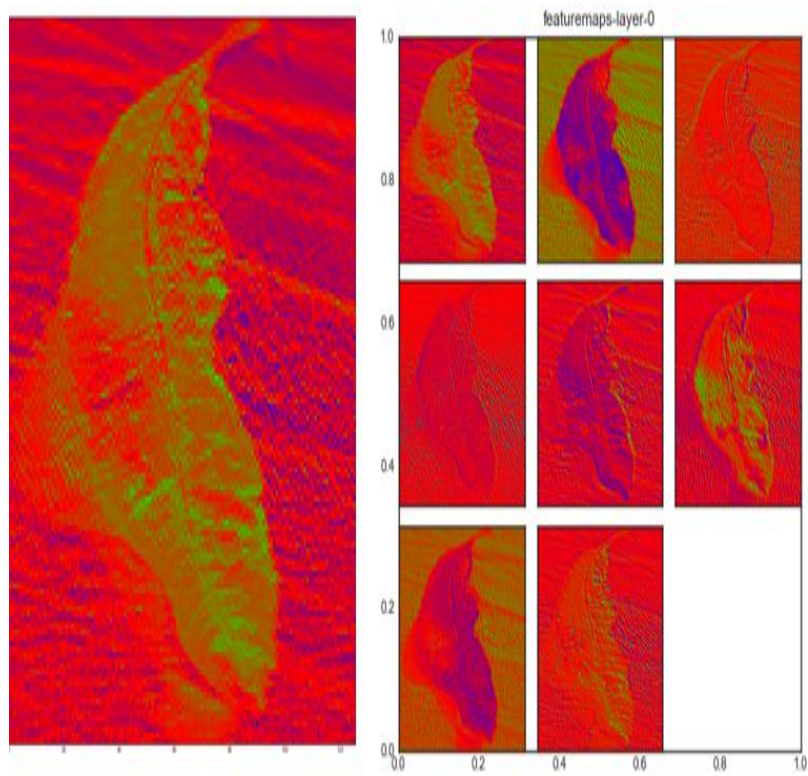

Figure 4: a) Feature maps 


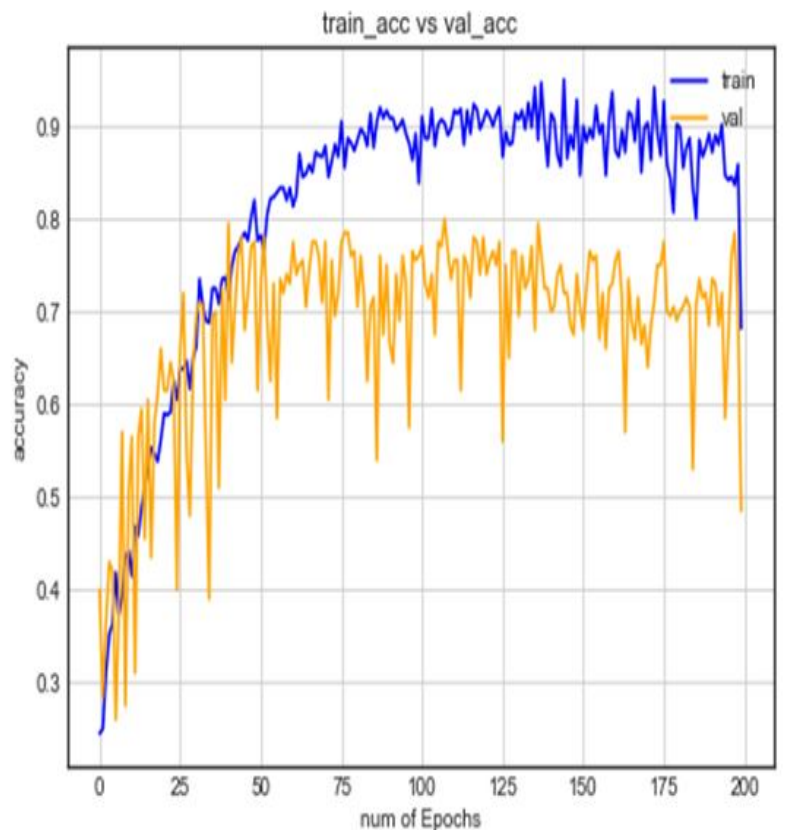

Figure 4 : b) Graphical representation of training accuracy vs epochs

Figure 4 a) The feature maps extricate the picture data; the feature map is the yield of one channel applied to the past layer. A given channel is drawn over the whole past layer, moved each pixel in turn. Each position brings about the enactment of the neuron and the yield is gathered in the element map. b) We had drawn the graph for the number of epoch's vs accuracy for training and testing of the data.

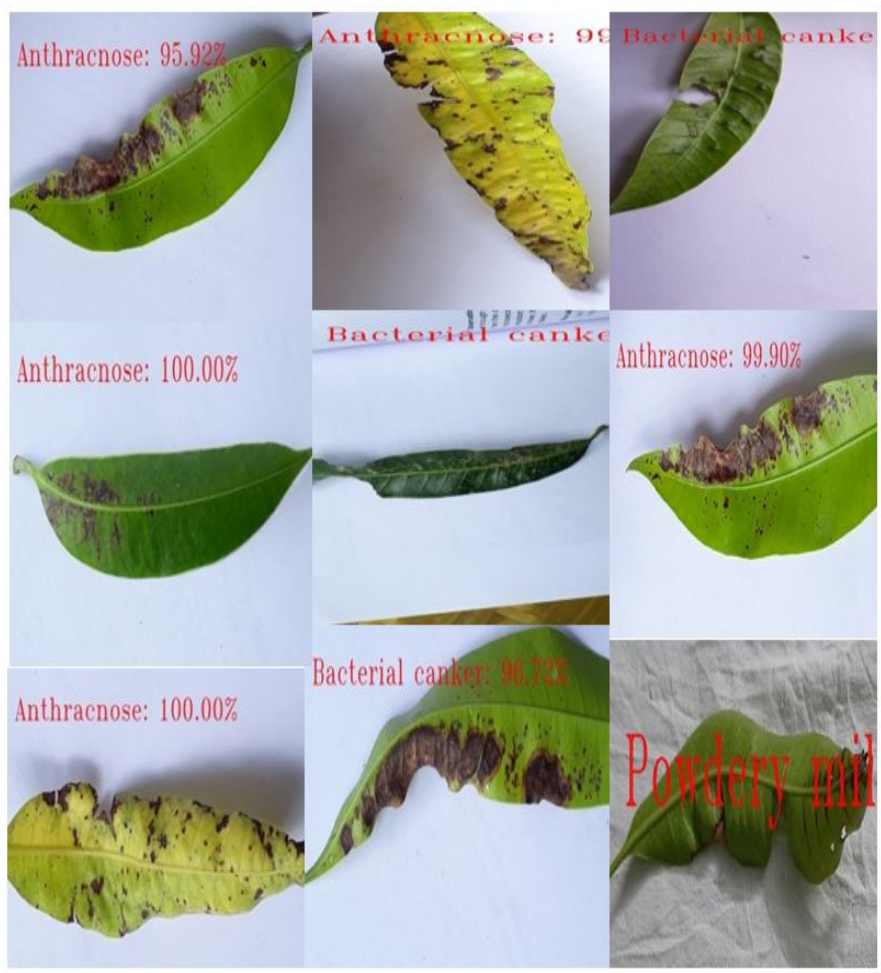

Figure 5: Accuracy obtained for the infected leaves
Figure $5 \mathrm{~s}$ shows the exactness acquired for each leaf we had tested a portion of the leaves got $100 \%$ precision, some got $90 \%$ precision. Anthracnose, powdery mildew, bacterial canker are the recognized sicknesses their precision normal is $96.72 \%$. The mismatch classification has happened for just a couple of leaves. The acquired after effect of our own proposed $\mathrm{CNN}$ model experiment for the leaves is appeared in the figure above.

\section{CONCLUSION}

This paper proposes the detection of diseases for the infected mango leaves. We selected the deep learning approach of our proposed design CNN which gives the average accuracy of $96.72 \%$ for batch 1 and $97.35 \%$ for batch 3 data. This deep learning technique extracts all features of the images and identifies the diseases of bacterial canker, anthracnose, and powdery mildew. The model used in CNN is single stride. A total of 800 pictures were taken and they were trained and tested 3 times like batch1, batch2, batch3. The results of recognition rates are mentioned in the table. 150 pictures are pre-trained for the system, 50 are tested to know the accuracy. In our proposed model we used Re-Lu as the activation function, convolution and max-pooling to extract the information regarding input image, dropout layers and dense layers are used to determine the output through the softmax layer. Our proposed model mainly helps the farmers to identify the disease that occurred to the leaves of the mango and can eradicate them in the initial state itself. So, the loss of harvesting crops for farmers can be reduced. Our project is completely a real-time approach mainly for the benefit of the farmers.

\section{REFERENCES}

1. P. Jiang, Y. Chen, B. Liu, D. He and C. Liang, Real-Time Detection of Apple Leaf Diseases Using Deep Learning Approach Based on Improved Convolutional Neural Networks, in IEEE Access, vol. 7, pp. 59069-59080, 2019. https://doi.org/10.1109/ACCESS.2019.2914929

2. X. Zhang, Y. Qiao, F. Meng, C. Fan and M. Zhang, "Identification of Maize Leaf Diseases Using Improved Deep Convolutional Neural Networks, in IEEE Access, vol. 6, pp. 30370-30377, 2018. https://doi.org/10.1109/ACCESS.2018.2844405

3. V. Sampath Kumar Reddy, M. Srimurali And B. Polaiah; Prediction of Pm10 And So2 Concentrations In Ambient Air Using Artificial Neural Networks For Hyderabad, International Journal of Civil Engineering (IJCE), Vol. 5, Issue 3, 2278-9995 Apr - May 2016; pp: $1-10$.

4. Umer Javed, etal; MRI and PET Image Fusion Using Fuzzy Logic and Image Local Features, The Scientific World Journal Volume No. 8, 2014 https://doi.org/10.1155/2014/708075 
5. Kumari, P.R.,, Polaiah Bojja, SO2 concentrations in the ambient air at vijayawada station using artificial neural networks computing, International Journal of Recent Technology and Engineering, 2019.

6. 193 .Rao G.A., Syamala K., Kishore P.V.V., Sastry A.S.C.S. .,Deep convolutional neural networks for sign language recognition, 2018, International Journal of Engineering and Technology(UAE), Vol: 7 ,Issue: 1.5 Special Issue 5 ,pp: 62 to:: 70 ,DOI: ,ISSN: $2227524 \mathrm{X}$

7. Raja kumari.P,.,A mathematical analysis of convective heat and mass transfer pour of a non -Newtonian fluid through porous medium in a rectangular duct with heat sources. Journal of Advanced Research in Dynamical and Control Systems, Volume 2017, Issue Special Issue 2, , Pages 84-91, 2017.

8. Polaiah Bojja, etal; Evaluation and Development of Advance Control Strategies Which Uses Pattern Recognition Technique for Nonlinear System, International Journal of Computer Theory and Engineering, Vol.3, No.1, February, 2011, pp: 1793-8201.

9. S. S. Chouhan, A. Kaul, U. P. Singh and S. Jain, Bacterial Foraging Optimization Based Radial Basis Function Neural Network (BRBFNN) for Identification and Classification of Plant Leaf Diseases: An Automatic Approach Towards Plant Pathology," in IEEE Access, vol. 6, pp. 8852-8863, 2018. https://doi.org/10.1109/ACCESS.2018.2800685

10. U. P. Singh, S. S. Chouhan, S. Jain and S. Jain, Multilayer Convolution Neural Network for the Classification of Mango Leaves Infected by Anthracnose Disease, in IEEE Access, vol. 7, pp. 4372143729, 2019.

https://doi.org/10.1109/ACCESS.2019.2907383

11. I. Pineda and O. Gwun, Leaf Modeling and Growth Process Simulation Using the Level Set Method, in IEEE Access, vol. 5, pp. 15948-15959, 2017.

12. Prasad M.V.D., Lakshmamma B.J., Chandana A.H., Komali K., Manoja M.V.N., Kumar P.R., Prasad C.R., Inthiyaz S., Kiran P.S. .,An efficient classification of flower images with convolutional neural networks , 2018, Lecture Notes in Electrical Engineering ,Vol: 434 ,Issue: ,pp: 419

13. Inthiyaz, Syed, B. T. P. Madhav, and P. V. V. Kishore. Flower segmentation with level sets evolution controlled by colour, texture and shape features. Cogent Engineering, vol.4, no. 1, pp.1-15, (2017): 1323572.

https://doi.org/10.1080/23311916.2017.1323572

14. Syed Inthiyaz, B. T. P Madhav, P. V. V. Kishore, Vamsi Krishna M., Sri Sai Ram Kumar M., Srikanth K. and Arun Teja B. Flower Image Segmentation: A Comparison Between Watershed, Marker Controlled Watershed, And Watershed Edge Wavelet Fusion . ARPN Journal of Engineering and Applied Sciences, vol.11, no.15, pp.9382-9387, (2016).

15. Ahammad, S. K. H., Rajesh, V., \& Ur Rahman, M. Z. (2019). Fast and accurate feature extraction based segmentation framework for spinal cord injury severity classification, IEEE Access, 7, 46092-46103. https://doi.org/10.1109/ACCESS.2019.2909583
16. 15. Syed Inthiyaz, B. T. P Madhav, P. V. V. Kishore, PreInformed Level Set for Flower Image Segmentation, International Conference on Smart Computing and Informatics (SCI), March 2017

17. 16. P. V. V. Kishore, M. V. D. Prasad, D. A. Kumar, and A. S. C. S. Sastry, Optical Flow Hand Tracking and Active Contour Hand Shape Features for Continuous Sign Language Recognition with Artificial Neural Networks, 2016 IEEE 6th International Conference on Advanced Computing (IACC), Feb. 2016.

18. 17. P. V. V. Kishore, D. A. Kumar, Goutham E.N.D, and M. Manikanta, Continuous sign language recognition from tracking and shape features using Fuzzy Inference Engine, 2016 International Conference on Wireless Communications, Signal Processing and Networking, Mar. 2016.

19. Rao, G. Ananth, and P. V. V. Kishore. Selfie video based continuous Indian sign language recognition system. Ain Shams Engineering Journal (2017). https://doi.org/10.1016/j.asej.2016.10.013

20. Bhavana D., Rajesh V., A new pixel level image fusion method based on genetic algorithm ,2016, Indian Journal of Science and Technology, Vol: 9, Issue: 45, pp: 1 - 8, ISSN 9746846 https://doi.org/10.17485/ijst/2016/v9i45/76691

21. Ahammad, S. H., Rajesh, V., Saikumar, K., Jalakam, S., \& Kumar, G. N. S. (2019). Statistical analysis of spinal cord injury severity detection on high dimensional MRI data. International Journal of Electrical and Computer Engineering, 9(5), 3457-3464

22. Reddy S.S., Suman M., Prakash K.N. Micro aneurysms detection using artificial neural networks , 2018, Lecture Notes in Electrical Engineering, Vol: 471, pp: 273. 